\title{
Game list colouring of graphs
}

\author{
M. Borowiecki \\ Faculty of Mathematics, Computer Science and Econometrics \\ University of Zielona Góra \\ Szafrana 4a, 65-516 Zielona Góra, Poland \\ m. borowiecki@wmie.uz.zgora.pl \\ E. Sidorowicz \\ Faculty of Mathematics, Computer Science and Econometrics \\ University of Zielona Góra \\ Szafrana 4a, 65-516 Zielona Góra, Poland \\ e.sidorowicz@wmie.uz.zgora.pl \\ Zs. Tuza* \\ Computer and Automation Institute \\ Hungarian Academy of Sciences, Hungary \\ and \\ Departament of Computer Science \\ Unversity of Pannonia, Veszprém, Hungary \\ tuza@sztaki.hu
}

Submitted: Jan 11, 2006; Accepted: Mar 15, 2007; Published: Mar 22, 2007

Mathematics Subject Classifications: 05C15, 05C75

\begin{abstract}
We consider the two-player game defined as follows. Let $(G, L)$ be a graph $G$ with a list assignment $L$ on its vertices. The two players, Alice and Bob, play alternately on $G$, Alice having the first move. Alice's goal is to provide an $L$-colouring of $G$ and Bob's goal is to prevent her from doing so. A move consists in choosing an uncoloured vertex $v$ and assigning it a colour from the set $L(v)$. Adjacent vertices of the same colour must not occur. This game will be called game list colouring. The game choice number of $G$, denoted by $\operatorname{ch}_{g}(G)$, is defined as the least $k$ such that Alice has a winning strategy for any $k$-list assignment of $G$.

We characterize the class of graphs with $\operatorname{ch}_{g}(G) \leq 2$ and determine the game choice number for some classes of graphs.
\end{abstract}

*Research supported in part by the Hungarian Scientific Research Fund, grant OTKA T-049613. 


\section{Introduction and notation}

All graphs considered in this paper are simple, i.e., finite, undirected, loopless and without multiple edges. The notation $(H \leq G) H \subseteq G$ means that $H$ is (an induced) subgraph of $G$. We say that $G$ contains $H$ whenever $G$ contains (an induced) subgraph isomorphic to $H$. The set of neighbours of a vertex $v \in V(G)$ is denoted by $N_{G}(v)$, or briefly by $N(v)$. The degree of $v$ is the number $|N(v)|$ and is denoted by $d(v)$. The symbol $\Delta(G)$ denotes the maximum degree of $G$. The vertex-disjoint union of two graphs $G$ and $H$ is denoted by $G \cup H$. By $P_{n}, C_{n}, S_{n}$ we denote the path, the cycle, the star with $n$ vertices. For undefined concepts we refer the reader to $[5,10]$.

Let $N$ denote the set of positive integers and $2^{N}$ denote the power set of $N$. Let $G=(V, E)$ be a graph. A function $f: V \rightarrow N$ is a colouring of $G$ if $f(v) \neq f(u)$ whenever $v u \in E$. A list assignment $L$ for $G$ is a function $L: V \rightarrow 2^{N}$. If $|L(v)|=k$ for every $v \in V$ then $L$ is a $k$-list-assignment for $G$. A graph $G$ with a given list assignment $L$ will be denoted by $(G, L)$.

A function $f: V \rightarrow N$ is an $L$-colouring of $G$ if $f$ is a colouring of $G$ with the additional requirement that $f(v) \in L(v)$ for all $v \in V(G)$. If $G$ admits an $L$-colouring, then $G$ is said to be $L$-colourable.

The graph $G$ is $k$-choosable if it is $L$-colourable for every $k$-list assignment. The choice number or the choosability of $G$, denoted by $\operatorname{ch}(G)$ is the smallest natural number $k$ such that $G$ is $k$-choosable.

The idea of considering $L$-colouring and $k$-choosability has been introduced independently by Vizing [18] and Erdős, Rubin and Taylor [6]. In both papers the choosability version of Brooks' theorem [3] was proved. The choosability version of Gallai's theorem [8] was proved independently by Thomassen [16] and by Kostochka et al. [14]. In [2] some extensions of these two basic theorems have been proved. A characterization of 2-choosable graphs is given in [6].

We consider the two-player game defined as follows. The two players are Alice and Bob and they play alternately with Alice having the first move. Given a graph $G$ and a set $C$ of colours, the players take turns colouring vertices of $G$ (one vertex per move) with colours from $C$. If after $|V(G)|$ moves the graph is coloured then Alice wins, otherwise Bob wins; i.e., Bob wins whenever an impasse is reached before the whole graph is coloured.

The game chromatic number of $G$, denoted by $\chi_{g}(G)$, is defined as the least cardinality of $C$ for which Alice has a winning strategy. The number $\chi_{g}(G)$ is well-defined since we have $\chi(G) \leq \chi_{g}(G) \leq|V(G)|$.

The game chromatic number of a graph was first introduced by Bodlaender in [1] where its computational complexity was studied. Bodlaender also showed that $\chi_{g}(T) \leq 5$ holds for all trees $T$, and exhibited trees with $\chi_{g}(T) \geq 4$. In [7] Bodlaender's upper bound was improved to $\chi_{g}(T) \leq 4$. Kierstead and Trotter proved in [12] that $\chi_{g}(G) \leq 33$ for every planar graph $G$ and that the maximum game chromatic number of an outerplanar graph is between 6 and 8. The currently known best upper bounds are 18 for planar graphs [11] and 7 for outerplanar graphs [9].

The edge-colouring version of this game was considered by Cai and Zhu [4]. Nešetřil 
and Sopena [15] introduced an oriented version of this game and developed some techniques for bounding the oriented game chromatic number. The conjecture of Nešetřil and Sopena was proved by Kierstead and Tuza in [13]. Further references on graph colouring games and $L$-colourings can be found e.g. in [17].

In this paper we are interested in another version of this game. We combine concepts of game and list colourings in the following way. Let $(G, L)$ be a graph $G$ with a list assignment $L$. Again the two players are Alice and Bob and they play alternately with Alice having the first move. Alice's goal is to provide an $L$-colouring of $G$ and Bob's goal is to prevent her from doing so. A move consists in choosing an uncoloured vertex $v$ and assigning it a colour from the set $L(v)$. This game will be called game list colouring.

We say that $(G, L)$ is game list colourable if Alice has a winning strategy. The game choice number of $G$, denoted by $\operatorname{ch}_{g}(G)$, is defined as the least $k$ such that Alice has a winning strategy for any $k$-list assignment of $G$. It is clear that this number is well-defined.

In Section 2 we present some basic properties of the game choice number and we investigate the relations between the game choice number, the game chromatic number and the choice number. In Section 3 we characterize the graphs whose game choice number is equal to two.

\section{Preliminary results}

From the definitions we immediately have the following relations:

$$
\begin{aligned}
\chi(G) & \leq \chi_{g}(G) \leq \operatorname{ch}_{g}(G) \leq \Delta(G)+1 \\
\chi(G) & \leq \operatorname{ch}(G) \leq \operatorname{ch}_{g}(G) \leq \Delta(G)+1
\end{aligned}
$$

The following result provides a sufficient condition for a graph with a list assignment $L$ to be $L$-colourable.

Theorem 1 [18, 6]. Let $G$ be a connected graph with a list assignment $L$ satisfying the following conditions:

(a) $|L(v)| \geq d(v)$ for every $v \in V(G)$,

(b) There is $v_{0} \in V(G)$ such that $\left|L\left(v_{0}\right)\right| \geq d\left(v_{0}\right)+1$.

Then $G$ is L-colourable.

Observation 1 Let $(G, L)$ be a graph with $|L(v)| \geq d(v)+1$ for every $v \in V(G)$. It is clear that $(G, L)$ is game list colourable, i.e., there is a winning strategy for Alice.

A natural question arises: Can Theorem 1 be extended to the game list colouring? In general the answer is NO. To explain this, we need some notation.

Let $(G, L)$ be a graph with $|L(v)| \geq d(v)$ for every $v \in V(G)$ and let

$$
\begin{gathered}
X_{(G, L)}=\{v \in V(G):|L(v)|=d(v)\} \\
Y_{(G, L)}=V-X_{(G, L)}=\{v \in V(G):|L(v)| \geq d(v)+1\} .
\end{gathered}
$$


Proposition 1 For any natural number $n$ there is a graph $(G, L)$ with $\left|Y_{(G, L)}\right|-\left|X_{(G, L)}\right|>$ $n$ such that $(G, L)$ is not game list colourable.

Proof. Let $\left(H, L_{1}\right)$ be a graph of even order, such that $\left|L_{1}(v)\right| \geq d(v)+1$ for all $v \in V(H)$. Let $\left(P_{4}, L_{2}\right)$ be the path with the list assignment $L_{2}(v)=\{1,2\}$ for all $v \in V\left(P_{4}\right)$. It is easy to see that Bob has a winning strategy on the graph $G=P_{4} \cup H$. Indeed, he can force Alice to be the first one who colours a vertex of the $P_{4}$ component; and then he can make $P_{4}$ uncolourable, by assigning the opposite colour at distance 2 from the firstly coloured one. If $|V(H)|=n+4$, then Proposition 1 follows.

$$
\text { Let } d_{(G, L)}=\min \left\{d(v): v \in X_{(G, L)}\right\} \text {. }
$$

Lemma 1 Let $(G, L)$ be a graph with $|L(v)| \geq d(v)$ for every $v \in V(G)$ and let $\left|X_{(G, L)}\right| \leq$ $\left\lceil\frac{d}{2}\right\rceil$, where $d=d_{(G, L)}$. Then Alice has a winning strategy.

Proof. Alice colours vertices of the set $X_{(G, L)}$ one by one until it gets completely coloured. It is possible because if $v$ is a vertex of $X_{(G, L)}$ which is coloured by Alice in the $k$-th move, then in the set $N(v)$ there are at most $k-1$ vertices coloured by Alice and at most $k-1$ vertices coloured by Bob. Since $2 k-2 \leq 2\left\lceil\frac{d}{2}\right\rceil-2<d(v)=|L(v)|$, Alice can choose a colour in $L(v)$ for the vertex $v$. Thus, the vertices of $X_{(G, L)}$ can be coloured from their lists. It is obvious (see Observation 1) that the vertices of $Y_{(G, L)}$ have a list colouring at any move of the game.

For complete bipartite graphs we have $\chi_{g}\left(K_{n, n}\right)=3(n \geq 2)$. From the next theorem it follows that for almost all complete bipartite graphs the game choice number is greater than the game chromatic number.

We will use the following notation. If Alice colours a vertex $v$ with colour $c$ at move $k$, then it will be denoted by $A_{k}:=[f(v)=c]$. Similarly, Bob's $k$-th move will be denoted by $B_{k}$.

Theorem 2 Let $n=\left(\begin{array}{c}k \\ \left\lfloor\frac{k+1}{2}\right\rfloor\end{array}\right)\left(\begin{array}{c}k \\ \left\lceil\frac{k+1}{2}\right\rceil\end{array}\right), \quad k \geq 2$. Then $\operatorname{ch}_{g}\left(K_{n, n}\right) \geq k+2$.

Proof. Let us denote the vertex classes of $K_{n, n}$ by $X=\left\{x_{1}, \ldots, x_{n}\right\}, Y=\left\{y_{1}, \ldots, y_{n}\right\}$. We split the $2 k$-element colour set $\{1,2, \ldots, 2 k\}$ into two disjoint $k$-sets $C_{1}=\{1,2, \ldots, k\}$ and $C_{2}=\{k+1, k+2, \ldots, 2 k\}$ and, as lists for the vertices, we take the $(k+1)$-subsets that are split equally or almost equally between $C_{1}$ and $C_{2}$. If $k+1$ is even, equal split is possible, each of the selected $(k+1)$-subsets will contain $\frac{k+1}{2}$ elements of $C_{1}$ and $\frac{k+1}{2}$ elements of $C_{2}$. If $k+1$ is odd, each of the selected $(k+1)$-subsets will contain $\frac{k}{2}+1$ elements of $C_{1}$ and $\frac{k}{2}$ elements of $C_{2}$. Assign each of these $(k+1)$-subsets of colours to one vertex of the set $X$ and to one vertex of the set $Y$.

To prove the theorem it is enough to find a winning strategy for Bob. We construct it as follows. By symmetry, assume that Alice colours in the first move a vertex of $X$ with a colour from its list, say $A_{1}:=\left[f\left(x_{1}\right)=c\right]$ and $c \in C_{i}(i=1$ or $i=2)$. During the first part of the game (at most $k$ moves of Bob), Bob will play on the set $X$ (because Alice started to colour a vertex of $X$ ). Bob's goal is to achieve a situation where at least $\left\lceil\frac{k+1}{2}\right\rceil$ 
colours of $C_{1}$ and $\left\lfloor\frac{k+1}{2}\right\rfloor$ colours of $C_{2}$ appear in $X$. If he can do so, then in the remaining part of the game the attempted choice by Alice must fail in the set $Y$ because the entire list of some vertex $y_{j} \in Y$ is excluded by the partial colouring constructed on $X$.

If $k$ is odd then Bob in the first move chooses an uncoloured vertex $x_{j}$ and assigns a colour from $L\left(x_{j}\right)$ which is not in $C_{i}$. If $k$ is even then Bob chooses for $x_{j}$ a colour from $C_{i}$. Having done so, an even number of non-used colours remain in both $C_{1}$ and $C_{2}$. Then, if in any one later step Alice colours a vertex of $Y$ with a colour $c \in C_{i}(i=1$ or $i=2)$ then Bob colours a vertex of $X$ which can be coloured with a new colour of the same $C_{i}$. Otherwise, if Alice colours a vertex of $X$ then Bob again chooses an uncoloured vertex of $X$ and assigns any new colour different than previously used by Alice and himself. The large number of vertices implies that Bob can follow this rule until more than half of $C_{1}$ and not less than half of $C_{2}$ appear in $X$. Thus $\left(K_{n, n}, L\right)$ is not $(k+1)$-game-list-colourable.

Let $G$ be the $r$-partite graph $K_{t_{1}, t_{2}, \ldots, t_{r}}$ such that $t_{1}=t_{2}=\ldots=t_{r}=2$. In [6] it has been shown that the choice number of $G$ is equal to $r$. It is easy to see that for this graph we have $\chi_{g}(G)=\operatorname{ch}_{g}(G)=\Delta(G)+1=2 r-1$, because Bob can repeat the colour assigned by Alice in each step, colouring the unique vertex nonadjacent to the latest choice of Alice. From the next theorem it follows that if one of the independent sets contains at least three vertices then $\operatorname{ch}_{g}\left(K_{t_{1}, t_{2}, \ldots, t_{r}}\right) \leq \Delta\left(K_{t_{1}, t_{2}, \ldots, t_{r}}\right)$.

Theorem 3 Let $r \geq 3,1 \leq i<r, t_{1}=t_{2}=\ldots=t_{i}=3, t_{i+1}=\ldots=t_{r}=2$. Then

$$
\operatorname{ch}_{g}\left(K_{t_{1}, t_{2}, \ldots, t_{r}}\right) \leq \Delta\left(K_{t_{1}, t_{2}, \ldots, t_{r}}\right) .
$$

Proof. Let $(G, L)$ be the $r$-partite graph that satisfies the assumptions of the theorem, with a $t$-list-assignment where $t=\Delta\left(K_{t_{1}, t_{2}, \ldots, t_{r}}\right)$. Let $V_{1}, V_{2}, \ldots, V_{r}$ be its independent sets and let $V_{1}=\left\{v_{1}, v_{2}, v_{3}\right\}$. We shall describe a winning strategy for Alice.

If there is a colour $c$ such that $c \in L\left(v_{1}\right) \cap L\left(v_{2}\right) \cap L\left(v_{3}\right)$ then $A_{1}:=\left[f\left(v_{1}\right)=c\right]$. In the second move Alice colours $v_{2}$ or $v_{3}$ with the colour $c$. Let $C$ be the set of coloured vertices after Bob's second move. The subgraph $G-C$ with the list assignment $L^{\prime}$ such that $L^{\prime}(v)=L(v) \backslash \bigcup_{u \in N(v) \cap C} f(u)$ for $v \in V(G-C)$ satisfies the assumptions of Lemma 1 and then Alice wins.

Suppose that $L\left(v_{1}\right) \cap L\left(v_{2}\right) \cap L\left(v_{3}\right)=\varnothing$. Then each colour of $L\left(v_{1}\right) \cup L\left(v_{2}\right) \cup L\left(v_{3}\right)$ is in at most two of the lists $L\left(v_{1}\right), L\left(v_{2}\right), L\left(v_{3}\right)$. Therefore Alice can choose a vertex $v_{i}$ such that $\left|L\left(v_{i}\right) \cap L\left(v_{j}\right)\right| \leq \frac{t}{2}$ for $i \neq j$. Assume that $v_{i}=v_{1}$. Let $V^{\prime} \subseteq V \backslash V_{1}$ be the set of vertices such that the subgraph induced by $V^{\prime}$ is the $(r-1)$-partite graph $K_{2,2, \ldots, 2}$. For any colour $c$ let us denote the vertex set $N(c)=\left\{v \in V^{\prime}: c \in L(v)\right\}$. In the first move Alice colours the vertex $v_{1}$ with the colour $c_{0}$ such that $\left|N\left(c_{0}\right)\right|=\min \left\{|N(c)|: c \in L\left(v_{1}\right)\right\}$. Suppose that $\left|N\left(c_{0}\right)\right| \leq r-1$. Let $v$ be the vertex coloured by Bob in the first move and $B_{1}:=[f(v)=c]$. Then the graph $\left(G-v_{1}\right)-v$ with the list assignment $L^{\prime}$ such that $L^{\prime}(w)=L(w) \backslash \bigcup_{u \in N(w) \cap C} f(u)$ for $w \in V\left(\left(G-v_{1}\right)-v\right)$ satisfies the assumptions of Lemma 1. Hence Alice has a winning strategy.

Assume that $\left|N\left(c_{0}\right)\right|>r-1$. Let us denote $\left|N\left(c_{0}\right)\right|=r+d$. We consider two cases: 
Case 1. In the first move Bob colours $v_{2}$ or $v_{3}$.

Assume that Bob colours $v_{2}$. If $c_{0} \in L\left(v_{3}\right)$ then Alice puts $A_{2}:=\left[f\left(v_{3}\right)=c_{0}\right]$. Let $C$ be the set of coloured vertices after Bob's second move. The subgraph $G-C$ with the list assignment $L^{\prime}$ such that $L^{\prime}(v)=L(v) \backslash \bigcup_{u \in N(v) \cap C} f(u)$ satisfies the assumptions of Lemma 1.

Suppose that $c_{0} \notin L\left(v_{3}\right)$. If there is $c \in L\left(v_{3}\right)$ such that $\left|N(c) \cap N\left(c_{0}\right)\right| \leq r-1$ then again by Lemma 1 Alice wins. We may assume that $\left|N(c) \cap N\left(c_{0}\right)\right| \geq r$ for $c \in L\left(V_{3}\right)$. We show that in this case $\sum_{v \in N\left(c_{0}\right)}|L(v)|>\left|N\left(c_{0}\right)\right| t$ which contradicts the assumption that each vertex has been assigned to a list of length $t$. By the choice of $c_{0},|N(c)| \geq r+d$ holds for all $c \in L\left(v_{1}\right)$. Then for any $c \in L\left(v_{1}\right)$ we have

$\sum_{v \in N\left(c_{0}\right)}|L(v)| \geq\left|N(c) \cap N\left(c_{0}\right)\right| \geq|N(c)|-\left|V^{\prime} \backslash N\left(c_{0}\right)\right| \geq r+d-2(r-1)+r+d \geq 2(d+1)$ and

$\sum_{v \in N\left(c_{0}\right)}\left|L(v) \cap \bigcup_{u \in V_{1}} L(u)\right| \geq \sum_{c \in L\left(v_{1}\right) \cup L\left(v_{3}\right)}\left|N(c) \cap N\left(c_{0}\right)\right| \geq$

$\sum_{c \in L\left(v_{3}\right)}\left|N(c) \cap N\left(c_{0}\right)\right|+\sum_{c \in L\left(v_{1}\right) \backslash L\left(v_{3}\right)}\left|N(c) \cap N\left(c_{0}\right)\right| \geq$

$r t+\left|L\left(v_{1}\right) \backslash L\left(v_{3}\right)\right| 2(d+1) \geq r t+2 \frac{t}{2}(d+1)>t(r+d)=\left|N\left(c_{0}\right)\right| t$,

a contradiction.

Case 2. In the first move Bob colours a vertex of $V-V_{1}$.

If $c_{0} \in L\left(v_{2}\right)$ or $c_{0} \in L\left(v_{3}\right)$ then by Lemma 1 Alice has a winning strategy. Assume that Bob colours the vertex $v^{\prime}$ and $B_{1}:=\left[f\left(v^{\prime}\right)=c^{\prime}\right]$. If $c^{\prime} \notin L\left(v_{2}\right)$ or $c^{\prime} \notin L\left(v_{3}\right)$ then similarly as in Case 1 we can show that there is a colour $c \in L\left(v_{2}\right)$ (or $c \in L\left(v_{3}\right)$ ) such that $\left|N(c) \cap N\left(c_{0}\right)\right|<r-1$. Then assume that $c^{\prime} \in L\left(v_{2}\right)$ and $c^{\prime} \in L\left(v_{3}\right)$ and, for all $c \in\left(L\left(v_{2}\right) \cup L\left(v_{3}\right)\right) \backslash\left\{c^{\prime}\right\},\left|N(c) \cap N\left(c_{0}\right)\right|>r-1$ holds. Then we have

$\sum_{v \in N\left(c_{0}\right)}\left|\left(L(v) \cap \bigcup_{u \in V_{1}} L(u)\right) \backslash\left\{c^{\prime}\right\}\right| \geq \sum_{v \in N\left(c_{0}\right)}\left|L(v) \cap \bigcup_{u \in V_{1}} L(u)\right|-\left|N\left(c_{0}\right)\right| \geq$

$\sum_{c \in L\left(v_{1}\right) \cup L\left(v_{3}\right)}\left|N(c) \cap N\left(c_{0}\right)\right|-\left|N\left(c_{0}\right)\right| \geq$

$\sum_{c \in L\left(v_{3}\right)}\left|N(c) \cap N\left(c_{0}\right)\right|+\sum_{c \in L\left(v_{1}\right) \backslash L\left(v_{3}\right)}\left|N(c) \cap N\left(c_{0}\right)\right|-\left|N\left(c_{0}\right)\right| \geq$

$r t+\left|L\left(v_{1}\right) \backslash L\left(v_{3}\right)\right| 2(d+1)-\left|N\left(c^{\prime}\right)\right|=t(r+d)+t-(r+d)$.

Recall that $r+d=\left|N\left(c_{0}\right)\right|$. Since $\left|N\left(c_{0}\right)\right| \leq 2(r-1)<t$, we have

$\sum_{v \in N\left(c_{0}\right)}\left|L(v) \cap \bigcup_{u \in V_{1}} L(u)\right| \geq \sum_{v \in N\left(c_{0}\right)}\left|\left(L(v) \cap \bigcup_{u \in V_{1}} L(u)\right) \backslash\left\{c^{\prime}\right\}\right|>t(r+d)=$ $\left|N\left(c_{0}\right)\right| t$, a contradiction.

\section{A characterization of graphs with game choice number two}

In this section we shall characterize the graphs for which Alice has a winning strategy in the game list colouring for any 2-list assignment.

The following proposition trivially holds.

Proposition 2 If $\operatorname{ch}_{g}(G)=2$ then $C_{2 k+1} \nsubseteq G$. 
Let us denote by $H_{1}$ and $H_{2}$ the 5 -vertex graphs exhibited in Figures 1 and 3, respectively.

Lemma 2 If $\operatorname{ch}_{g}(G)=2$ then $G$ does not contain any graph of the set $\left\{H_{1}, P_{5}, K_{2,3}, H_{2}\right\}$ as an induced subgraph.

Proof. Suppose that $G$ contains an induced subgraph $H$ isomorphic to $H_{1}$. Let us denote the vertices of $H$ as in Figure 1. Let $L$ be the 2-list-assignment such that $L(v)=\{1,2\}$ for $v \in V(H)$ and $L(u)=\{3,4\}$ for $u \in V(G)-V(H)$. We will give a winning strategy for Bob. If in the first move Alice colours a vertex $v$ of $H$ then Bob colours the vertex $w$ of $H$ such that $N_{H}(v) \cap N_{H}(w) \neq \varnothing$ using another colour than Alice did. Thus a third colour is necessary to colour the common neighbour of $v$ and $w$. If in the first move Alice colours a vertex $v$ of $G-H$ then Bob chooses the vertex $x_{1}$ of $H$ and he puts $B_{1}:=\left[f\left(x_{1}\right)=1\right]$. After the second move of Alice, Bob colours a vertex $w \in V(H)$ with $N_{H}(w) \cap N_{H}(u)=\varnothing$ ( $u$ is a vertex coloured by Alice or Bob) using a colour different from the colour of $u$. Such $w$ can be chosen, no matter which vertex has been coloured by Alice (e.g., if Alice has coloured $x_{5}$, then Bob can consider $u=x_{1}$ and $w=x_{2}$ ). Then a third colour is necessary to colour the common uncoloured neighbour of $w$ and $u$.

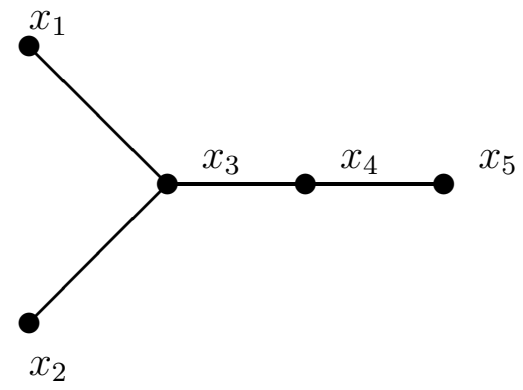

Figure 1. The graph $H_{1}$

Suppose that $G$ contains an induced subgraph $P$ isomorphic to $P_{5}$. Let $V(P)=$ $\left\{x_{1}, x_{2}, x_{3}, x_{4}, x_{5}\right\}$ and $E(P)=\left\{x_{1} x_{2}, x_{2} x_{3}, x_{3} x_{4}, x_{4} x_{5}\right\}$. Let $L$ be the 2-list-assignment such that $L(v)=\{1,2\}$ for all $v \in V(P)$ and $L(u)=\{3,4\}$ for all $u \in V(G)-V(P)$. If Alice colours $x_{i}$ with colour $j \in\{1,2\}$, the next move of Bob is to colour $x_{i-2}$ or $x_{i+2}$ with $3-j$. Otherwise, if Alice colours a vertex of $G-P$, Bob puts colour 1 on $x_{3}$, and then the colour 2 on $x_{1}$ or $x_{5}$ in his next move.

Suppose that $G$ contains an induced subgraph $K$ isomorphic to $K_{2,3}$. Let us denote the vertices of $K$ as in Figure 2. Let $L$ be the 2-list-assignment such that $L(v)=\{4,5\}$ for $v \in V(G)-V(K)$ and as given in Figure 2 for the vertices of $K$. Suppose that in the first move Alice colours a vertex $v$ of $K$. Then Bob chooses the vertex $w$ such that $N_{H}(v) \cap N_{H}(w) \neq \varnothing$. It is easy to see that Bob can colour $w$ from its list such that the common neighbour of $v$ and $w$ cannot be coloured. Now, suppose that in the first move Alice colours a vertex $v$ of $G-K$. Then Bob's move is $B_{1}:=\left[f\left(x_{1}\right)=1\right]$. This leaves the path $x_{3} x_{2} x_{4}$ with the reduced lists $\{3\},\{3,4\},\{4\}$, which is not list-colourable, no matter how Alice and Bob play afterward. 


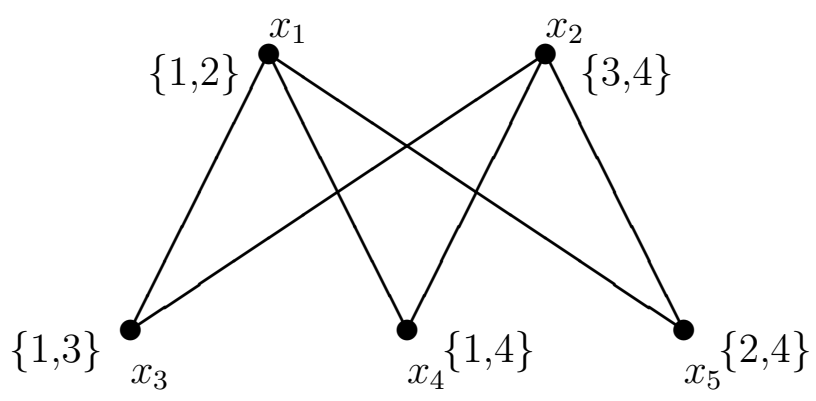

Figure 2. The 2-list-assignment of $K_{2,3}$

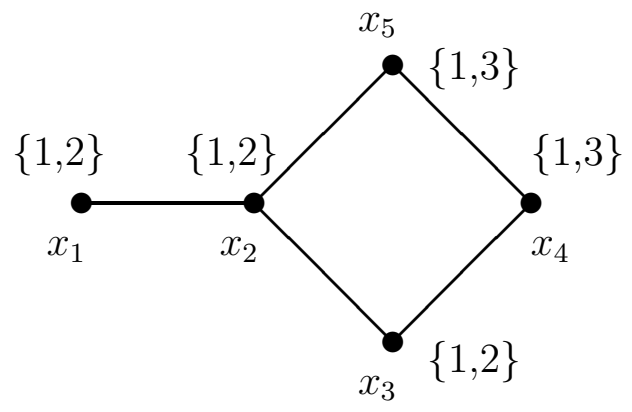

Figure 3. The 2-list-assignment of $\mathrm{H}_{2}$

Suppose that $G$ contains an induced subgraph $H$ isomorphic to $H_{2}$. Let us denote the vertices of $H$ and its 2-list-assignment $L$ as in Figure 3 and let $L(v)=\{3,4\}$ for $v \in V(G)-V(H)$. Assume that in the first move Alice colours a vertex of $H$. We may assume that Alice colours neither $x_{1}$ nor $x_{3}$, otherwise Bob can make a move after which the vertex $x_{2}$ cannot be coloured. Suppose that Alice chooses the vertex $x_{2}$. If she puts $A_{1}:=\left[f\left(x_{2}\right)=1\right]$ then Bob answers $B_{1}:=\left[f\left(x_{4}\right)=3\right]$. After such moves the vertex $x_{5}$ cannot be coloured from its list. If $A_{1}:=\left[f\left(x_{2}\right)=2\right]$ then $B_{1}:=\left[f\left(x_{4}\right)=1\right]$. Thus the vertex $x_{3}$ cannot be coloured. Similarly we can show that in the first move Alice cannot choose to colour the vertex $x_{4}$. If in the first move Alice colours $x_{5}$ with colour 1 then Bob colours the vertex $x_{1}$ with 2. If Alice puts $A_{1}:=\left[f\left(x_{5}\right)=3\right]$ then Bob's move is $B_{1}:=\left[f\left(x_{3}\right)=1\right]$. Hence a third colour is necessary to colour the vertex $x_{4}$. If in the first move Alice colours a vertex of $G-H$ then Bob's move is $B_{1}:=\left[f\left(x_{1}\right)=1\right]$. If in the second move Alice does not colour the vertex $x_{2}$ or $x_{3}$ then the second Bob's move is $B_{2}:=\left[f\left(x_{3}\right)=2\right]$. Hence the vertex $x_{2}$ cannot be coloured, but if the second move of Alice is $A_{2}:=\left[f\left(x_{2}\right)=2\right]$ then Bob chooses $B_{2}:=\left[f\left(x_{4}\right)=1\right]$. If $A_{2}:=\left[f\left(x_{3}\right)=1\right]$ then Bob chooses $B_{2}:=\left[f\left(x_{5}\right)=3\right]$. In both cases after Bob's second move there is a vertex in $V(H)$, which cannot be coloured.

The next corollary is an immediate consequence of Lemma 2.

Corollary 1 If $\operatorname{ch}_{g}(G)=2$ then each component of $G$ is isomorphic to $P_{4}$ or $C_{4}$, or $S_{k}$ for some $k \geq 1$. 
Let in the process of the game list colouring the graph $G$ be partially coloured. We introduce the following notation. Let $C$ be the set of coloured vertices and $U$ be the set of uncoloured vertices. For any vertex $v$ we will denote $N_{C}(v)=N(v) \cap C$ and $N_{U}(v)=N(v) \cap U$. We will say that the vertex $x$ is safe if $x$ is uncoloured and $\mid L(x)-$ $\bigcup_{u \in N_{C}(x)} f(u)|>| N_{U}(x) \mid$.

The definition immediately yields the following

Proposition 3 Suppose that in the process of the game list colouring a graph $G$ is partially coloured. If all uncoloured vertices of $G$ are safe then Alice wins.

Now we will consider a game list colouring but with the first move of Bob. The rest principles of the game are the same: Two players Alice and Bob play alternately but Bob has the first move. Alice's goal is to provide a proper list colouring of the graph and Bob's goal is to prevent her from doing so. We shall refer to this version as B-game list colouring. The original version, i.e. Alice moving first, will sometimes be called the A-game.

If Alice has a winning strategy on $H$ in the B-game then in some situations she can use it for finding a winning strategy on a larger graph $G$, which contains $H$ such that $|V(G) \backslash V(H)|$ is odd. Let $k$ be an integer and $G$ be a graph such that $G=G_{1} \cup G_{2}$ and $\left|V\left(G_{1}\right)\right|$ is odd. If Alice has a winning strategy in the A-game on $G_{1}$ with $k$ colours and she also has a winning strategy with $k$ colours in the B-game on $G_{2}$ then she can obtain a winning strategy on $G$ in the following way: First Alice marks the vertex of $G_{1}$ according to her winning strategy on $G_{1}$. After that she plays on $G_{i}(i=1$ or $i=2)$ if and only if Bob has played on $G_{i}$ in his previous move. In each move she follows her winning strategy in the A-game or B-game, according as $i=1$ or $i=2$.

Lemma 3 Alice has a winning strategy in the B-game list colouring on $P_{4}$.

Proof. Let $V\left(P_{4}\right)=\left\{x_{1}, x_{2}, x_{3}, x_{4}\right\}$ and $E\left(P_{4}\right)=\left\{x_{1} x_{2}, x_{2} x_{3}, x_{3} x_{4}\right\}$. Bob has the first move.

Suppose that Bob's move is $B_{1}:=\left[f\left(x_{1}\right)=a\right]$. Alice chooses vertex $x_{3}$. If $L\left(x_{2}\right) \neq$ $L\left(x_{3}\right)$ then Alice colours the vertex $x_{3}$ with the colour from $L\left(x_{3}\right)$ which is not in $L\left(x_{2}\right)$. After this move all vertices of $P_{4}$ are safe; then by Proposition 3 Alice wins. Now assume that $L\left(x_{2}\right)=L\left(x_{3}\right)$. If $a \in L\left(x_{3}\right)$ then $A_{1}:=\left[f\left(x_{3}\right)=a\right]$. Otherwise Alice can colour $x_{3}$ with any colour of $L\left(x_{3}\right)$ and after her move all vertices of $P_{4}$ are safe.

If Bob's move is $B_{1}:=\left[f\left(x_{2}\right)=a\right]$ then Alice colours $x_{3}$ using another colour than Bob did. Then all vertices of $P_{4}$ are safe. The lemma follows.

Since $\operatorname{ch}_{g}\left(P_{4}\right)=3$, we also have

Corollary 2 If $\operatorname{ch}_{g}(G)=2$ and $P_{4} \leq G$ then $\left|V(G)-V\left(P_{4}\right)\right|$ is odd.

Lemma 4 Let $\left(C_{4}, L\right)$ be the 4-cycle with the 2-list-assignment $L$ as in Figure 4 or Figure 5. Then Alice has a winning strategy on $\left(C_{4}, L\right)$ in the A-game and Bob has a winning strategy on $\left(C_{4}, L\right)$ in the B-game. 
Proof. First let us consider A-game, Alice has the first move and $A_{1}:=\left[f\left(x_{2}\right)=a\right]$. If Bob colours the vertex $x_{1}$ or $x_{4}$ then after his move all vertices of the graph are safe. If Bob colours the vertex $x_{3}$ using the colour $b$ or $c$ then Alice colours $x_{1}$ with $d$, thus after her move the vertex $x_{4}$ can be coloured with $a$.

Now assume that Bob starts. It is easy to see that Bob can win, as in the first move he can colour the vertex $x_{1}$ using colour $a$ and then the rest is uncolourable.

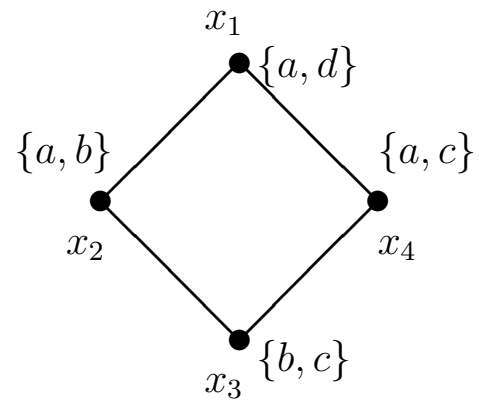

Figure 4.

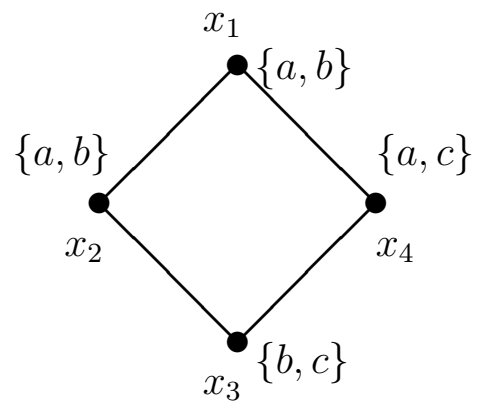

Figure 5 .

Corollary 3 If $\operatorname{ch}_{g}(G)=2$ then $G$ does not contain two vertex-disjoint $C_{4} s$.

Let $G$ be a graph with a subgraph isomorphic to $C_{4}$ and with a 2-list-assignment $L$. From Lemma 4 it follows that there are 2-list-assignments of $C_{4}$ such that in a winning strategy of Alice, she must start choosing a vertex of $C_{4}$. But there are also 2-listassignments such that Alice wins if and only if in the process of the game list colouring Bob first colours a vertex of $C_{4}$.

Lemma 5 Let $L$ be a 2-list-assignment of $C_{4}$, other than the lists in Figure 4 and Figure 5. Then Alice has a winning strategy in the B-game list colouring on $\left(C_{4}, L\right)$.

Proof. Let $V\left(C_{4}\right)=\left\{x_{1}, x_{2}, x_{3}, x_{4}\right\}, E\left(C_{4}\right)=\left\{x_{1} x_{2}, x_{2} x_{3}, x_{3} x_{4}, x_{4} x_{1}\right\}$. Without loss of generality assume that Bob chooses $x_{1}$ and puts $B_{1}:=\left[f\left(x_{1}\right)=a\right]$. If $a \in L\left(x_{3}\right)$ then Alice colours $A_{1}:=\left[f\left(x_{3}\right)=a\right]$. Hence all vertices are safe and Alice wins. Then assume that $a \notin L\left(x_{3}\right)$ and $L\left(x_{3}\right)=\{b, c\}$. If neither $A_{2}:=\left[f\left(x_{3}\right)=b\right]$ nor $A_{2}:=\left[f\left(x_{3}\right)=c\right]$ leaves both $x_{2}$ and $x_{4}$ colourable (i.e., if Alice cannot win by colouring $x_{3}$ ), then their lists must be $L\left(x_{2}\right)=\{a, b\}$ and $L\left(x_{4}\right)=\{a, c\}$, or vice versa. Thus, the list assignment is isomorphic to the one in Figure 4 or 5 , depending on whether the second element in $L\left(x_{1}\right)$ is a new colour or one of $b$ and $c$.

Corollary 4 If $\operatorname{ch}_{g}(G)=2$ and $C_{4} \leq G$ then $\left|V(G)-V\left(C_{4}\right)\right|$ is odd.

Since Alice has a winning strategy on any star forest in both the A-game and B-game list colourings, we obtain the main result of this section.

Theorem 4 Let $G$ be a graph with at least one edge. Then $\operatorname{ch}_{g}(G)=2$ if and only if $G$ is a star forest or $G=k P_{4} \cup t C_{4} \cup H$, where $k \geq 0, t \in\{0,1\}$ and $H$ is a star forest of odd order. 


\section{References}

[1] H.L. Bodlaender, On the complexity of some coloring games, Internat. J. Found. Comput. Sci. 2 (1991) 133-147.

[2] M. Borowiecki, E. Drgas-Burchardt and P. Mihók Generalized list colourings of graphs, Discussiones Math. Graph Theory 15 (1995) 185-193.

[3] R.L. Brooks, On colouring the nodes of a network, Proc. Cambridge Phil. Soc. 37 (1941) 194-197.

[4] L. Cai and X. Zhu, Game chromatic index of k-degenerate graphs, J. Graph Theory 36 (2001) 144-155.

[5] G. Chartrand and L. Lesniak, Graphs and Digraphs, Second Edition, (Wadsworth \& Brooks/Cole, Monterey, 1986).

[6] P. Erdős, A.L. Rubin and H. Taylor, Choosability in graphs, in: Proc. West Coast Conf. on Combin., Graph Theory and Computing, Congressus Numerantium XXVI (1979) 125-157.

[7] U. Faigle, U. Kern, H.A. Kierstead and W.T. Trotter, On the game chromatic number of some classes of graphs, Ars Combinatoria 35 (1993) 143-150.

[8] T. Gallai, Kritische Graphen II, Magyar Tud. Akad. Mat. Kut. Int. Közl. 8 (1963) 373-395.

[9] D.J. Guan and X. Zhu, Game chromatic number of outerplanar graphs, J. Graph Theory 30 (1999) 67-70.

[10] T.R. Jensen and B. Toft, Graph Colouring Problems, (Wiley, New York, 1995).

[11] H.A. Kierstead, A simple competitive graph coloring algorithm, J. Combin. Theory B 78 (2000) 57-68.

[12] H.A. Kierstead and W.T. Trotter, Planar graph coloring with an uncooperative partner, J. Graph Theory 18 (1994) 569-584.

[13] H.A. Kierstead and Zs. Tuza, Marking games and the oriented game chromatic number of partial k-trees, Graphs and Combinatorics 19 (2003) 121-129.

[14] A.V. Kostochka, M. Stiebitz and B. Wirth, The colour theorems of Brooks and Gallai extended, Discrete Math. 162 (1996) 299-303.

[15] J. Nešetřil and E. Sopena, On the oriented game chromatic number, Electronic J. Combin. 8 (No. 2) (2001) \#R14.

[16] C. Thomassen, Color-critical graphs on a fixed surface, J. Combin. Theory B 70 (1997) 67-100.

[17] Zs. Tuza, Graph colorings with local constraints - A survey, Discuss. Math. Graph Theory 17 (1997) 161-228.

[18] V.G. Vizing, Colouring the vertices of a graph in prescribed colours, Diskret. Analiz 29 (1976) 3-10 (in Russian). 